\title{
A note on Lovász removable path conjecture
}

\author{
JIE MA
}

\begin{abstract}
Lovász [8] conjectured that for any natural number $k$, there exists a smallest natural number $f(k)$ such that, for any two vertices $s$ and $t$ in any $f(k)$-connected graph $G$, there exists an $s$ - $t$ path $P$ such that $G-V(P)$ is $k$-connected. This conjecture is proved only for $k \leq 2$. Here, we strengthen the result for $k=2$ as follows: for any integers $l>0$ and $m \geq 0$, there exists a function $f(l, m)$ such that, for any distinct vertices $s, t, v_{1}, \ldots, v_{m}$ in any $f(l, m)$ connected graph $G$, there exist $l$ internally vertex disjoint $s$ - $t$ paths $P_{1}, \ldots, P_{l}$ such that for any subset $I \subset\{1, \ldots, l\}, G-\cup_{i \in I} V\left(P_{i}\right)$ is 2-connected and $\left\{v_{1}, v_{2}, \ldots, v_{m}\right\} \subset V(G)-\cup_{1 \leq i \leq l} V\left(P_{i}\right)$.
\end{abstract}

\section{Introduction}

The following conjecture is due to Lovász [8] which is still open for $k \geq 3$ :

Conjecture 1.1. For any natural number $k$, there exists a least natural number $f(k)$ such that, for any two vertices $s, t$ in any $f(k)$-connected graph $G$, there exists an s-t path $P$ such that $G-V(P)$ is $k$-connected.

This conjecture has been proved for $k \leq 2$. A theorem of Tutte [11] shows that $f(1)=3$. When $k=2$, we have $f(2)=5$ by a result of Chen, Gould and $\mathrm{Yu}[2]$ and, independently, of Kriesell [6]. Later, Kawarabayashi, Lee and $\mathrm{Yu}$ [4] characterized the 4-connected graphs $G$ in which there exist two vertices $s, t \in V(G)$ such that $G-V(P)$ is not 2-connected for any $s$ - $t$ path $P$ in $G$.

Conjecture 1.1 is equivalent to asking whether there exists a function $g(k)$ such that for any $g(k)$-connected graph and for any edge $s t \in E(G)$, there exists a cycle $C$ containing st such that $G-V(C)$ is $k$-connected. Lovász [8] also made a weaker conjecture: any $(k+3)$-connected graph contains a cycle $C$ such that $G-V(C)$ is $k$-connected, which was confirmed by Thomassen [10]. Another weaker version of Conjecture 1.1 was proposed by Kriesell [7]: there exists a function $h(k)$ such that for any $h(k)$-connected graph $G$ and for any two vertices $s, t \in V(G)$, there exists an induced $s$ - $t$ path $P$ in $G$ such that $G-E(P)$ is $k$-connected. This weaker version was 
established by Kawarabayashi, Lee, Reed and Wollan [3]. In [3], the authors further conjectured that there exists a function $F(k)$ such that for any $F(k)$-connected graph $G$ and for any three distinct vertices $s, t, u \in V(G), G$ contains an $s$ - $t$ path $P$ and a $k$-connected subgraph $H$ such that $u \in V(H)$ and $V(H) \cap V(P)=\emptyset$; they also show that this conjecture implies Conjecture 1.1. In this sense, it is useful to find an $s$ - $t$ path that avoids a highly connected subgraph containing a specific vertex, which partially motivates our work.

Conjecture 1.1 asks for one removable path. In [2], Chen, Gould and Yu showed that in any $(22 l+2)$-connected graph, there exist $l$ internally vertex disjoint paths between any two given vertices such that the deletion of any one of these paths results in a connected graph. Recently, Kawarabayashi and Ozeki [5] strengthened this result as follows: for any $(3 l+2)$-connected graph $G$ and for any two vertices $s, t \in V(G)$, there exist $l$ internally vertex disjoint $s$ - $t$ paths $P_{1}, \ldots, P_{l}$ such that $G-\cup_{i=1}^{l} V\left(P_{i}\right)$ is 2-connected; they also pointed out that if $G$ is $(2 l+1)$-connected, then one can find $l$ internally vertex disjoint paths $P_{1}, \ldots, P_{l}$ between any two given vertices such that $G-\cup_{i=1}^{l} V\left(P_{i}\right)$ is connected.

In this note, we use a short argument to prove the following:

Theorem 1.2. For any integers $l>0$ and $m \geq 0$, let $f(l, m)=30 l+10 m+2$. Then for any distinct vertices $s, t, v_{1}, \ldots, v_{m}$ in any $f(l, m)$-connected graph $G$, there exist $l$ internally vertex disjoint $s$-t paths $P_{1}, \ldots, P_{l}$ such that for any subset $I \subset\{1, \ldots, l\}, G-\cup_{i \in I} V\left(P_{i}\right)$ is 2 -connected and $\left\{v_{1}, v_{2}, \ldots, v_{m}\right\} \subset$ $V(G)-\cup_{1 \leq i \leq l} V\left(P_{i}\right)$.

\section{Proof of Theorem 1.2}

We begin with some definitions. A linkage is a graph in which every connected component is a path. A linkage problem in a graph $G$ is a set of pairs of vertices of $G$, for example, $\mathcal{L}=\left\{\left\{s_{1}, t_{1}\right\}, \ldots,\left\{s_{k}, t_{k}\right\}\right\}$. A solution to the linkage problem $\mathcal{L}$ is a set of pairwise internally vertex disjoint paths $P_{1}, \ldots, P_{k}$ such that the ends of $P_{i}$ are $s_{i}$ and $t_{i}$, and if $x \in V\left(P_{i}\right) \cap V\left(P_{j}\right)$ for $i \neq j$ then $x=s_{i}$ or $x=t_{i}$. The graph $G$ is $k$-linked if every linkage problem with $k$ pairwise disjoint pairs of vertices has a solution.

Bollobás and Thomason [1] proved that every 22k-connected graph is $k$ linked. Here we use the following improved bound by Thomas and Wollan [9]:

Lemma 2.1. Every $10 k$-connected graph is $k$-linked.

We also need the following lemma. 
Lemma 2.2. For any distinct vertices $s_{1}, \ldots, s_{l}, t_{1}, \ldots, t_{l}, v_{1}, \ldots, v_{m}$ in $(30 l+10 m)$-connected graph $G$, there exist $l$ internally vertex disjoint paths $P_{1}, \ldots, P_{l}$ in $G$ and a 2-connected subgraph $H$ of $G-\cup_{1 \leq i \leq l} V\left(P_{i}\right)$ such that the ends of $P_{i}$ are $s_{i}$ and $t_{i}$ for $1 \leq i \leq l,\left\{v_{1}, \ldots, v_{m}\right\} \subset V(H)$, $|V(H)| \geq 2 l+m$, and every vertex in $\left\{s_{1}, \ldots, s_{l}, t_{1}, \ldots, t_{l}\right\}$ has at least one neighbor in $H$.

Proof. We may find a neighbor $a_{i}$ of $s_{i}$ and a neighbor $b_{i}$ of $t_{i}$, for $1 \leq i \leq l$, such that $a_{1}, \ldots, a_{l}, b_{1}, \ldots, b_{l}, s_{1}, \ldots, s_{l}, t_{1}, \ldots, t_{l}, v_{1}, \ldots, v_{m}$ are pairwise distinct, since $G$ is $(30 l+10 m)$-connected. Now we look at the following linkage problem in $G$ :

$$
\begin{aligned}
\mathcal{L}=\{ & \left\{s_{1}, t_{1}\right\}, \ldots,\left\{s_{l}, t_{l}\right\},\left\{a_{1}, a_{2}\right\},\left\{a_{2}, a_{3}\right\}, \ldots,\left\{a_{l}, b_{1}\right\}, \\
& \left.\left\{b_{1}, b_{2}\right\}, \ldots,\left\{b_{l}, v_{1}\right\},\left\{v_{1}, v_{2}\right\}, \ldots,\left\{v_{m}, a_{1}\right\}\right\}
\end{aligned}
$$

which has $3 l+m$ pairwise disjoint pairs of vertices. By Lemma 2.1, we have a solution of $\mathcal{L}$ : a collection of $3 l+m$ paths $\left\{P_{1}, \ldots, P_{3 l}, Q_{1}, \ldots, Q_{m}\right\}$, where, for $1 \leq i \leq 3 l+m$, the ends of the $i$ th path of this collection (in the order listed) are the two vertices of the $i$ th pair in $\mathcal{L}$ (in the order listed). Let $H=\left(\cup_{l+1 \leq i \leq 3 l} P_{i}\right) \cup\left(\cup_{1 \leq j \leq m} Q_{j}\right)$, which is a cycle through $a_{1}, \ldots, a_{l}, b_{1}, \ldots, b_{l}, v_{1}, \ldots, v_{m}$. Note that $|V(H)| \geq 2 l+m$. Then $P_{1}, \ldots, P_{l}$ and $H$ satisfy the conclusion of the lemma.

Now, we are ready to give the proof of Theorem 1.2 .

Proof. We may assume that $l \geq 2$ or $m \geq 1$; otherwise, $l=1, m=0$ and the theorem follows from known results. So we have that $l+m \geq 2$. Let $G^{\prime}=G-\{s, t\}$. Since $G$ is $(30 l+10 m+2)$-connected, $G^{\prime}$ is $(30 l+10 m)$ connected. We may fix $l$ neighbors of $s$, say $s_{1}, s_{2}, \ldots, s_{l}$, and $l$ neighbors of $t$, say $t_{1}, t_{2}, \ldots, t_{l}$, such that $s_{1}, \ldots, s_{l}, t_{1}, \ldots, t_{l}, v_{1}, \ldots v_{m}$ are distinct.

By Lemma 2.2, there is a collection $\mathscr{P}=\left\{P_{1}, \ldots, P_{l}\right\}$ of paths in $G^{\prime}$ such that $\left\{v_{1}, \ldots, v_{m}\right\}$ is contained in a 2-connected subgraph $D(\mathscr{P})$ of $G^{\prime}-\cup_{i=1}^{l} V\left(P_{i}\right),|V(D(\mathscr{P}))| \geq 2 l+m$ and any vertex of $\left\{s_{1}, \ldots, s_{l}, t_{1}, \ldots, t_{l}\right\}$ has a neighbor in $D(\mathscr{P})$. We call such collection $\mathscr{P}$ feasible. We may choose $D(\mathscr{P})$ to be a maximal 2-connected subgraph of $G^{\prime}-\cup_{1 \leq i \leq l} V\left(P_{i}\right)$, and if there is no ambiguity we simply call it $D$. Without loss of generality, we assume that the ends of $P_{i}$ are $s_{i}$ and $t_{i}$ for any $1 \leq i \leq l$. If $D=$ $G^{\prime}-\cup_{1 \leq i \leq l} V\left(P_{i}\right)$, then $\left\{s, s s_{1}\right\} \cup P_{1} \cup\left\{t_{1} t, t\right\}, \ldots,\left\{s, s s_{l}\right\} \cup P_{l} \cup\left\{t_{l} t, t\right\}$ satisfy the conclusion of Theorem 1.2. Thus we may assume $D \neq G^{\prime}-\cup_{1 \leq i \leq l} V\left(P_{i}\right)$, and let $C_{1}, \ldots, C_{q}$ be the components of $G^{\prime}-\cup_{1 \leq i \leq l} V\left(P_{i}\right)-V(\bar{D})$. By the 
maximality of $D, D$ contains at most one neighbor of $V\left(C_{i}\right)$ for $1 \leq i \leq q$. Without loss of generality, we assume that

$$
\left|V\left(C_{1}\right)\right| \geq\left|V\left(C_{2}\right)\right| \geq \cdots \geq\left|V\left(C_{q}\right)\right| .
$$

We choose a feasible collection $\mathscr{P}=\left\{P_{1}, \ldots, P_{l}\right\}$ in $G^{\prime}$ such that

(1) $|V(D(\mathscr{P}))|$ is maximum, and

(2) subject to (1), $\left|V\left(C_{1}\right)\right|,\left|V\left(C_{2}\right)\right|, \ldots,\left|V\left(C_{q}\right)\right|$ are as large as possible with the larger order components having priority, and

(3) subject to $(2),\left|V\left(\cup_{1 \leq i \leq l} P_{i}\right)\right|$ is as small as possible.

Note that by Lemma $2.2,|V(D(\mathscr{P}))| \geq 2 l+m$. Now we consider $G^{0}:=$ $G^{\prime}\left[\left(\cup_{1 \leq i \leq l} P_{i}\right) \cup C_{q}\right]$. We claim that there exist a subset $J \subset\{1,2, \ldots, l\}$ and $\left\{a_{j}, b_{j}\right\} \subset V\left(P_{j}\right)$ for all $j \in J$ such that $G^{\prime}\left[\left(\cup_{j \in J} a_{j} P_{j} b_{j}\right) \cup C_{q}\right]$ is connected and it is separated from the other vertices of $G^{0}$ by $\left\{a_{j}, b_{j}: j \in J\right\}$. The existence of $J$ follows by taking $G^{\prime}\left[\left(\cup_{j \in J} a_{j} P_{j} b_{j}\right) \cup C_{q}\right]$ to be the component of $G^{0}$ containing $C_{q}$. Without loss of generality, we assume that $b_{j} \in a_{j} P_{j} t_{j}$ for $j \in J$ (possible $a_{j}=b_{j}$ ). We pick $J,\left\{a_{j}, b_{j}: j \in J\right\}$ such that

(4) if $J^{\prime} \subset J$ and $\left\{a_{j}^{\prime}, b_{j}^{\prime}\right\} \subset V\left(a_{j} P_{j} b_{j}\right)$ for $j \in J^{\prime}$ are such that $G^{\prime}\left[C_{q} \cup\right.$ $\left.\left(\cup_{j \in J^{\prime}} a_{j}^{\prime} P_{j} b_{j}^{\prime}\right)\right]$ is connected and separated from the other vertices of $G^{0}$ by $\left\{a_{j}^{\prime}, b_{j}^{\prime}: j \in J^{\prime}\right\}$, then $J^{\prime}=J$ and for $j \in J, a_{j}^{\prime}=a_{j}$ and $b_{j}^{\prime}=b_{j}$.

In this sense, we call $J,\left\{a_{j}, b_{j}: j \in J\right\}$ minimal. We may assume that $J=$ $\{1, \ldots, r\}, r \leq l$. Let $G^{1}:=G^{\prime}\left[\left(\cup_{j \in J} a_{j} P_{j} b_{j}\right) \cup C_{q}\right]$, and $N_{q}:=V(D) \cap N\left(C_{q}\right)$ (hence $\left|N_{q}\right| \leq 1$ ). We will prove the following claim, for any $k \in J$ and any $x, y \in V\left(a_{k} P_{k} b_{k}-\left\{a_{k}, b_{k}\right\}\right)$, where $y \in x P_{k} b_{k}-\left\{b_{k}\right\}$ (possible $x=y$ ).

Claim. There exist $r$ vertex disjoint paths in $G^{1}-V\left(x P_{k} y\right)$ from $A:=\left\{a_{j}\right.$ : $1 \leq j \leq r\}$ to $B:=\left\{b_{j}: 1 \leq j \leq r\right\}$.

Proof of Claim. Without loss of generality, we say $k=1$. If not, then by Menger's Theorem there exists a cut of size $p \leq r-1$ in $G^{1}-V\left(x P_{1} y\right)$, say $W:=\left\{w_{2}, w_{3}, \ldots, w_{p+1}\right\}$, separating $A$ from $B$. We see that $a_{j} P_{j} b_{j}$ has at least one vertex in $W$ for $2 \leq j \leq r$; otherwise $a_{j} P_{j} b_{j}$ connects $A$ and $B$. Thus $p=r-1$ and we may assume that $w_{j} \in V\left(a_{j} P_{j} b_{j}\right)$ for $2 \leq j \leq r$. Now, $W \cup V\left(x P_{1} y\right)$ is a cut in $G^{1}$ which separates $A$ from $B$.

Let $D_{1}=\left(\left(\cup_{2 \leq j \leq r} a_{j} P_{j} w_{j}\right) \cup a_{1} P_{1} x\right)-(W \cup\{x\}), D_{2}=\left(\left(\cup_{2 \leq j \leq r} w_{j} P_{j} b_{j}\right) \cup\right.$ $\left.y P_{2} b_{1}\right)-(W \cup\{y\})$. We point out that at most one of $\left\{D_{1}, D_{2}\right\}$ contains a neighbor of $C_{q}$; otherwise, we can find a path in $G^{1}$ from $A$ to $B$ through $C_{q}$, disjoint from $W \cup V\left(x P_{1} y\right)$, contradicting to the fact that $W \cup V\left(x P_{1} y\right)$ is a cut in $G^{1}$ separating $A$ from $B$. Without loss of generality, we assume 
that $D_{1}$ does not contain any neighbor of $C_{q}$. Thus $W \cup V\left(x P_{1} y\right)$ separates $A$ from $C_{q} \cup B$.

We may assume that $x \neq y$. Otherwise we have $x=y$, then $W \cup\{x\}$ separates $A$ from $C_{q} \cup B$, but $x \in V\left(a_{1} P_{1} b_{1}-\left\{a_{1}, b_{1}\right\}\right)$, so it contradicts (4), in particular the choice of $A$. Now, we consider $G^{2}:=G^{\prime}\left[\left(\cup_{2 \leq j \leq r} a_{j} P_{j} w_{j}\right) \cup\right.$ $\left.a_{1} P_{1} y\right]$, and contract $x P_{1} y-\{x\}$ into a new vertex $x^{\prime}$, then call the resulting graph $G^{3}$. Note that $x x^{\prime}$ is an edge in $G^{3}$.

There exist $r$ vertex disjoint paths from $A$ to $W \cup\left\{x^{\prime}\right\}$ in $G^{3}-\{x\}$. Otherwise, by Menger's Theorem, there is a cut of size $t \leq r-1$ in $G^{3}-\{x\}$, say $W^{\prime}=\left\{w_{2}^{\prime}, \ldots, w_{t+1}^{\prime}\right\}$, separating $A$ from $W \cup\left\{x^{\prime}\right\}$. Clearly, $a_{j} P_{j} w_{j}$ has at least one vertex in $W^{\prime}$ for $2 \leq j \leq r$; so $t=r-1$ and we may assume that $w_{j}^{\prime} \in V\left(a_{j} P_{j} w_{j}\right)$ for $2 \leq j \leq r$. Then, it means that $W^{\prime} \cup\{x\}$ separates $A$ from $W \cup V\left(x P_{1} y\right)$ in $G^{2}$; since $W \cup V\left(x P_{1} y\right)$ separates $A$ from $C_{q} \cup B$ in $G^{1}, W^{\prime} \cup\{x\}$ separates $A$ from $C_{q} \cup B$ in $G^{1}$. But $x \in V\left(a_{1} P_{1} b_{1}\right)-\left\{a_{1}, b_{1}\right\}$, which contradicts (4), in particular $W^{\prime} \cup\{x\}$ contradicts the choice of $A$.

Therefore, there exist $r$ vertex disjoint paths in $G^{2}-\{x\}$ from $A$ to $W \cup\{u\}$, for some $u \in V\left(x P_{1} y\right)-\{x\}$, say $P_{1}^{\prime}$ from $a_{\pi(1)}$ to $u$ and $P_{j}^{\prime}$ from $a_{\pi(j)}$ to $w_{j}$ for $2 \leq j \leq r$, where $\pi$ is a permutation of $\{1, \ldots, r\}$. Then, we have a new collection $\mathscr{P}^{\prime}=\left\{P_{1}^{\prime}, \ldots, P_{l}^{\prime}\right\}$, where $P_{1}^{\prime}=s_{\pi(1)} P_{\pi(1)} a_{\pi(1)} \cup$ $a_{\pi(1)} P_{1}^{\prime} u \cup u P_{1} t_{1}, P_{i}^{\prime}=s_{\pi(i)} P_{\pi(i)} a_{\pi(i)} \cup a_{\pi(i)} P_{i}^{\prime} w_{i} \cup w_{i} P_{i} t_{i}$ for $2 \leq i \leq r$ and $P_{j}^{\prime}=P_{j}$ for $r+1 \leq j \leq l$. We see that $\mathscr{P}^{\prime}$ is a feasible collection of $G^{\prime}$ and satisfies (1) and (2), but $V\left(\cup_{1 \leq i \leq l} P_{i}^{\prime}\right) \subset V\left(\cup_{1 \leq i \leq l} P_{i}\right)-\{x\}$, which contradicts (3).

Let $N:=N\left(G^{1}-A \cup B\right)-V\left(G^{1}\right)$. By the choice of $J$, we see that $N \subset \cup_{i=1}^{q-1} V\left(C_{i}\right) \cup V(D)$.

We may assume that $N \subset V(D)$. If not, there exists $C_{h}$ such that $1 \leq$ $h \leq q-1$ and $V\left(C_{h}\right) \cap N \neq \emptyset$, then $x \in N\left(C_{h}\right) \cap V\left(a_{k} P_{k} b_{k}-\left\{a_{k}, b_{k}\right\}\right) \neq \emptyset$ for some $k \in J$. By Claim, there exist $r$ vertex disjoint paths in $G^{1}-\{x\}$ from $A$ to $B$, say $a_{\pi(j)} P_{j}^{\prime} b_{j}, 1 \leq j \leq r$, where $\pi$ is a permutation of $\{1, \ldots, r\}$. Then we have a new collection $\mathscr{P}^{\prime}=\left\{P_{1}^{\prime}, \ldots, P_{l}^{\prime}\right\}$, where $P_{i}^{\prime}=s_{\pi(i)} P_{\pi(i)} a_{\pi(i)} \cup$ $a_{\pi(i)} P_{i}^{\prime} b_{i} \cup b_{i} P_{i} t_{i}$ for $1 \leq i \leq r$ and $P_{j}^{\prime}=P_{j}$ for $r+1 \leq j \leq l$. We see that $\mathscr{P}^{\prime}$ is a feasible collection in $G^{\prime}$, such that $V\left(\cup_{1 \leq i \leq l} P_{i}^{\prime}\right) \subset V\left(\cup_{1 \leq i \leq l} P_{i} \cup C_{q}\right)-\{x\}$, then $\mathscr{P}^{\prime}$ either contradicts (1) or satisfies (1) but contradicts $(2)$.

We may assume that there exists $k \in J$ such that $\mid V(D) \cap N\left(a_{k} P_{k} b_{k}-\right.$ $\left.\left\{a_{k}, b_{k}\right\}\right) \mid \geq 2$. Otherwise, for any $j \in J$, we have $\mid V(D) \cap N\left(a_{j} P_{j} b_{j}-\right.$ $\left.\left\{a_{j}, b_{j}\right\}\right) \mid \leq 1$; since $N=V(D) \cap N=\cup_{j \in J}\left(V(D) \cap N\left(a_{j} P_{j} b_{j}-\left\{a_{j}, b_{j}\right\}\right)\right)$, we have $|N| \leq r \leq l$. Note that $|V(D)| \geq 2 l+m$ and $l+m \geq 2$, so $\left|V\left(D-N \cup N_{q}\right)\right| \geq 2 l+m-l-1 \geq 1$, which means $D-N \cup N_{q} \neq \emptyset$. Note that $N \cup A \cup B \cup N_{q}$ is a cut of $G^{\prime}$ separating $C_{q}$ from $D-N \cup N_{q}$, but 
$\left|V\left(N \cup A \cup B \cup N_{q}\right)\right| \leq 3 l+1$, contradicting to the $(30 l+10 m)$-connectedness of $G^{\prime}$.

Let $\left\{v_{1}, v_{2}\right\} \subset V(D) \cap N\left(a_{k} P_{k} b_{k}-\left\{a_{k}, b_{k}\right\}\right)$ and $\{x, y\} \subset V\left(a_{k} P_{k} b_{k}-\right.$ $\left.\left\{a_{k}, b_{k}\right\}\right)$ such that $v_{1} \neq v_{2}, v_{1} x \in E(G), v_{2} y \in E(G), y \in x P_{k} b_{k}-\left\{b_{k}\right\}$. By claim, there exist $r$ vertex disjoint paths in $G^{1}-V\left(x P_{k} y\right)$ from $A$ to $B$, say $a_{\pi(j)} P_{j}^{\prime} b_{j}, 1 \leq j \leq r$, where $\pi$ is a permutation of $\{1, \ldots, r\}$. Then we have a new collection $\mathscr{P}^{\prime}=\left\{P_{1}^{\prime}, \ldots, P_{l}^{\prime}\right\}$, where $P_{i}^{\prime}=s_{\pi(i)} P_{\pi(i)} a_{\pi(i)} \cup a_{\pi(i)} P_{i}^{\prime} b_{i} \cup$ $b_{i} P_{i} t_{i}$ for $1 \leq i \leq r$ and $P_{j}^{\prime}=P_{j}$ for $r+1 \leq j \leq l$. We see that $\mathscr{P}^{\prime}$ is a feasible collection in $G^{\prime}$, such that $V\left(\cup_{1 \leq i \leq l} P_{i}^{\prime}\right) \subset V\left(\cup_{1 \leq i \leq l} P_{i} \cup C_{q}\right)-V\left(x P_{k} y\right)$. But $\mathscr{P}^{\prime}$ contradicts $(1)$, since $D(\mathscr{P}) \cup V\left(x P_{k} y\right) \subset D\left(\mathscr{P}^{\prime}\right)$. This completes the proof of Theorem 1.2.

\section{Concluding remarks}

We note that in Theorem 1.2, those $l$ internally vertex disjoint $s$-t paths $P_{1}, \ldots, P_{l}$ are not induced; but we can strengthen the result by asking $P_{i}-$ $\{s, t\}$ be induced for all $1 \leq i \leq l$. The function $f(l, m)=30 l+10 m+2$ is likely not optimal since we use the result that $10 k$-connected graph is $k$-linked, and $10 k$ is not known to be optimal for the $k$-linkage problem. It is easy to see that an improvement on the $k$-linkage problem will give us a better function $f(l, m)$. We point out that a similar argument (after slight modification) gives a different and shorter proof of the theorem in [5] mentioned in Section 1.

Our result motivates us to propose the following question:

Question. For any integers $k, l>0$ and $m \geq 0$, there exists a function $f(k, l, m)$ such that the following holds. For any distinct vertices $s, t, v_{1}, \ldots$, $v_{m}$ in any $f(k, l, m)$-connected graph $G$, there exist $l$ internally vertex disjoint $s$ - $t$ paths $P_{1}, \ldots, P_{l}$ such that for any subset $I \subset\{1, \ldots, l\}, G-$ $\cup_{i \in I} V\left(P_{i}\right)$ is $k$-connected and $\left\{v_{1}, v_{2}, \ldots, v_{m}\right\} \subset V(G)-\cup_{1 \leq i \leq l} V\left(P_{i}\right)$.

We see that when $l=1, m=0$ this question is equivalent to Conjecture 1.1, and Theorem 1.2 shows that this question is true when $k=2$.

\section{Acknowledgements}

I would especially like to thank Xingxing Yu for helpful discussion and valuable comments. 


\section{References}

[1] Bollobás, B. and Thomason, A. (1996). Highly linked graphs. Combinatorica 16 313-320. MR1417341

[2] Chen, G., Gould, R., and Yu, X. (2003). Graph connectivity after path removal. Combinatorica 23 185-203. MR2001907

[3] Kawarabayashi, K., Lee, O., Reed, B., and Wollan, P. (2008). A weaker version of Lovász' path removable conjecture. J. Combin. Theory, Ser. B 98 972-979. MR2442591

[4] Kawarabayashi, K., Lee, O., and Yu, X. (2005). Non-separating paths in 4-connected graphs. Ann. Comb. 9(1) 47-56. MR2135775

[5] Kawarabayashi, K. and Ozeki, K. (2011). Non-separating subgraphs after deleting many disjoint paths. J. Combin. Theory, Ser. B 101(1) 54-59. MR2737178

[6] Kriesell, M. (2001). Induced paths in 5-connected graphs. J. Graph Theory 36 52-58. MR1803633

[7] Kriesell, M. Removable paths conjectures, http://www.fmf.unilj.si/ mohar/Problems/P0504Kriesell1.pdf.

[8] Lovász, L. (1975). Problems in Recent Advances in Graph Theory, (ed. M. Fiedler), Academia, Prague. MR0363962

[9] Thomas, R. and Wollan, P. (2005). An improved linare edge bound for graph linkage. European J. Combin. 26 309-324. MR2116174

[10] Thomassenb, C. (1981). Non-separating cycles in $k$-connected graphs. J. Graph Theory 5 351-354. MR0635696

[11] Tutte, W. T. (1963). How to draw a graph. Proc. London Math. Soc. 13 743-767. MR0158387

JIE MA

SCHOOL OF Mathematics

Georgia Institute of Technology

Atlanta, GA 30332-0160

USA

E-mail address: jiema@math.gatech.edu

RECEIVED June 6, 2010 